\title{
Analysing self-regulated learning strategies of MOOC learners through self-reported data
}

\author{
M. Elena Alonso-Mencía, Carlos Alario-Hoyos, Iria Estévez-Ayres, Carlos Delgado Kloos \\ Department of Telematics Engineering, Universidad Carlos III de Madrid, Spain
}

\begin{abstract}
Massive open online courses (MOOCs) require registered learners to be autonomous in their learning. Nevertheless, prior research studies showed that many learners lack the necessary self-regulated learning (SRL) skills to succeed in MOOCs. This research study aimed to gain insights into the relationships that exist between SRL and background information from MOOC learners. To this end, a series of three MOOCs on computer programming offered through edX were used to collect self-reported data from learners using an adaptation of the Motivated Strategies for Learning Questionnaire. Results show significant differences in general learning strategies and motivation by continent, prior computing experience and percentage of completed MOOCs. Men reported higher motivation than women, whereas pre-university learners needed further guidance to improve their learning strategies.

\section{Implications for practice:}

- A better understanding of learners' SRL strategies can help design better MOOCs.

- An early identification of learners with poor SRL strategies can help to reduce dropout rates of those learners who actually intend to finish the MOOC.

- A better understanding of differences between groups of students with respect to their SRL strategies can help design MOOCs with personalised variations for a better performance.
\end{abstract}

Keywords: MOOCs, self-regulated learning, learning strategies, motivation, self-reported survey, programming

\section{Introduction}

Massive open online courses (MOOCs) have gained popularity since 2012. Nevertheless, despite the high number of learners registered in MOOCs, most related research publications report completion rates between 5\% and 15\% (Clow, 2013; Davis et al., 2017). This phenomenon, called funnel of participation (Clow, 2013), is considered almost inevitable in any MOOC. Some reasons learners provide for dropping out of a MOOC include the course structure (Onah et al., 2014), low motivation (Kornell \& Finn, 2016), isolation feelings (Khalil \& Ebner, 2014), poor study techniques (Eriksson et al., 2017), or lack of selfregulated learning (SRL) skills such as self-efficacy (Halawa et al., 2014) or time management (Eriksson et al., 2017). The cultural context of learners, and its relationship with SRL, is also relevant when analysing dropout in MOOCs (Bozkurt \& Akbulut, 2019). Interestingly, not all reasons for leaving a MOOC early are necessarily negative, as some learners simply drop out once they have learned what they wanted to or needed (Eriksson et al., 2017).

The importance of self-regulation in this particular learning environment is well known (Halawa et al., 2014; Kornell \& Finn, 2016) as learners are required to be autonomous and they have great freedom to choose their learning path. However, some studies found that many MOOC learners lack the necessary SRL skills to undertake a MOOC (Alario-Hoyos et al., 2017; Eriksson et al., 2017). Some researchers have recommended interventions to support learners who lack the appropriate SRL skills, as MOOC platforms "lack technological assistance" (Pérez-Álvarez et al., 2017). Gašević et al. (2014) called for more research regarding SRL in MOOCs. In fact, the number of publications in this area has increased since 2016 (Lee et al., 2019), mainly through exploratory studies (Alonso-Mencía et al., 2020). Furthermore, authors have conducted research to better understand learners' profiles and ensure their engagement throughout the learning experience in MOOCs (e.g., Guo \& Reinecke, 2014; Kizilcec et al., 2016).

The aim of this research study was to contribute to this line of research and to gain insights into the SRL skills of the learners registered in a series of three MOOCs on programming offered in edX, analysing possible relationships between the characteristics of these learners and their SRL skills. Results from this 
research study will be useful for researchers or instructors who are interested in identifying learners at risk (with difficulties to complete the course) to offer them additional support. Our research questions (RQ) were as follows:

- RQ1: Are there significant differences on self-reported SRL skills among demographic groups?

- RQ2: Are there significant differences on self-perceived motivation and learning strategies according to prior computing experience?

- RQ3: Are there significant differences on learners' SRL skills according to previous MOOC completion percentage?

\section{Related literature}

SRL is an umbrella term under which many variables are studied, as it involves cognitive, metacognitive, motivational, behavioural and affective processes (Panadero, 2017). Due to its complexity, several models have been proposed in the literature to summarise all the aspects involved in SRL process (Pintrich, 2000b; Zimmerman, 2000). Despite their different conceptualisations, most authors conceive SRL as a cyclic threephase process: preparatory, performance and appraisal (Panadero, 2017; Puustinen \& Pulkkinen, 2001). The preparatory phase includes SRL strategies such as goal setting and strategic planning and is highly conditioned by the motivation and beliefs of the learner, including aspects such as goal orientation, task value, outcome expectations or self-efficacy. The performance phase includes SRL strategies such as time management, study environment management, help seeking; during this phase both cognitive and metacognitive processes take place. The appraisal phase includes SRL strategies such as self-reflection, self-evaluation or self-satisfaction; during this phase, metacognitive processes mainly take place.

SRL was first studied in traditional face-to-face environments (Hood et al., 2015), concluding that good SRL skills are linked to better academic achievement (Zimmerman, 2002; Zimmerman \& Pons, 1986). This result also occurs in online environments (Barnard-Brak et al., 2010). Kocdar et al. (2018) developed an instrument for measuring self-regulation in self-paced open and distance learning environments; they pointed out that there is a need for more research on SRL in online environments but including different cultural settings, as is the case in MOOCs. Unlike most other online courses, MOOCs offer more flexibility to learners because they have the freedom to choose and follow their own learning path. In order to succeed in a MOOC, it is crucial for learners to be able to develop a set of SRL strategies to self-manage their learning process and achieve their goals (Maldonado-Mahauad et al., 2018). For example, learners with good goal setting and strategic planning skills are more likely to achieve their goals in a MOOC (Kizilcec et al., 2017).

A recent systematic literature review on SRL and MOOCs identified the need for more interventions in this area (Alonso-Mencía et al., 2020). Specifically, this literature review found 29 exploratory studies and only 13 evaluations of interventions. In the evaluations of interventions, 3 subgroups were identified: use of external tools (e.g., Pérez-Álvarez et al., 2017), MOOC interventions or path recommendations (e.g., Jo et al., 2016) and face-to-face study group proposals (e.g., Chen \& Chen, 2015). Exploratory research studies identified in this literature review were more diverse. Researchers tried to find out more about learners' SRL skills, and their relationship, for example, to learners' characteristics or motivation (Kizilcec et al., 2017), attitude (Watson et al., 2017), course design (Campbell et al., 2014; Watson et al., 2017) or engagement (Phan et al., 2016).

Demographic characteristics appear to be an influential factor in the SRL skills of MOOC learners. For example, Kizilcec et al. (2017) found that SRL strategies differ by gender in MOOC environments, unlike findings in previous studies on technology-enhanced learning (Liou \& Kuo, 2014). Although women reported higher scores on goal setting, task value and help-seeking, men stood out in strategic planning, elaboration and self-evaluation. In addition, Guo and Reinecke (2014) found that older learners followed a non-linear navigation across the MOOC, which is associated with stronger SRL skills, because these older learners preferred to create their own learning paths. This behaviour was also found in learners from countries with lower numbers of students per teacher, although the influence of age was stronger. Moreover, Kizilcec et al. (2017) concluded that learners with higher education levels consistently reported stronger SRL skills, except for help-seeking, in line with findings in previous studies (Hood et al., 2015). Differences were also found with respect to learners' background (Hood et al., 2015) and their familiarity with MOOCs 
(Kizilcec et al., 2017).

SRL is closely related to motivation (Pintrich, 2000a). De Barba et al. (2016) found that motivation was an important predictor of performance in MOOCs, and its lack was a relevant reason for dropping out (Eriksson et al., 2017). Motivation also usually has a direct relationship with SRL skills in MOOC environments, as learners who self-reported being motivated, also reported higher SRL skills (Littlejohn et al., 2016). However, motivation may change and is influenced by the MOOC context (De Barba et al., 2016). Keeping learners motivated throughout the course might reduce dropouts of those learners who actually intend to complete the MOOC. Interestingly, Kizilcec et al. (2017) stated that learners could be trained to engage in SRL through motivation. For instance, if learners are motivated and feel confident in the learning environment, their SRL skills will improve (Pintrich, 2000a). Our research delved into the learner characteristics or factors that may help more efficiently to identify differences in SRL skills and motivation levels, in the context of MOOCs. Insights from the study may help instructors recognise groups at risk of dropout and provide them with the adequate mechanisms to support them.

\section{Methodology}

\section{Research design}

A survey research methodology (Creswell, 2002) was applied, taking a sample of individual MOOC learners. This was a cross-sectional quantitative study, as the data were collected via an online survey at a certain point in time. Given that the survey was optional, it is important to take into account the possible bias of the data collected especially in view of the high number of non-respondents. The survey had two parts:

- Part 1: Background questions. Learners were asked about their age, gender, country, educational level, and previous experience with MOOCs, computing or programming.

- Part 2: Questionnaire on SRL skills. The questionnaire was based on the well-known instrument developed by Pintrich et al. (1991): Motivated Strategies for Learning Questionnaire (MSLQ). However, it required some adaptation to the MOOC context, as MSLQ is designed for a classroom environment. In the adapted questionnaire, both motivation and learning strategies sections are included, which comprised a subset of MSLQ scales and items (see Table $1 \&$ Appendix). The adapted questionnaire consisted of 3 scales in the motivation block: intrinsic goal orientation (4 items), task value (6 items) and self-efficacy for learning and performance ( 8 items); and 2 scales in the learning strategies block: time and study environment ( 7 items), and critical thinking (5 items). All the items were assessed by the learners on a scale from 1 (minimum value) to 7 (maximum value).

Table 1

Scales and items of the questionnaire included in the survey

\begin{tabular}{llc}
\hline Section & \multicolumn{1}{c}{ Scale } & Number of items \\
\hline Motivation & Intrinsic goal orientation & 4 \\
& Task value & 6 \\
& Self-efficacy for learning and performance & 8 \\
\hline Learning strategies & Time and study environment & 7 \\
& Critical thinking & 5 \\
\hline
\end{tabular}

\section{Variables}

This subsection presents the variables under study, consisting of dependent and independent variables (see Table 2). The survey research aimed to find learner subgroups with difficulties to self-regulate their learning, particularly low motivation and poor learning strategies. For that purpose, the relationship between a dependent variable and one or several independent variables was studied.

Dependent variables

The dependent variables are the items selected from the adapted version of the MSLQ (7-point Likert scales per item). According to the manual developed by Pintrich et al. (1991) on the use of the MSLQ, the mean 
score for each scale must be calculated. There are 2 learning strategies scales and 3 motivation scales. The scales (which are all continuous) are:

- Time and study environment (7 items). Time management refers to learners' skills to schedule, plan, organise and manage their learning time in the MOOC successfully. Study environment refers to the conditions of the physical environment where learners study for the MOOC.

- Critical thinking (5 items). This scale refers to learners' ability to apply previous knowledge to make critical evaluations of the presented concepts in the MOOC.

- Intrinsic goal orientation (4 items). This scale refers to learners' goals and reasons to engage in the MOOC, such as challenge or curiosity.

- Task value (6 items). This scale refers to learners' opinions on the tasks (how interesting, useful or important the MOOC is for the learner).

- $\quad$ Self-efficacy for learning and performance (8 items). This scale refers to learners' performance expectations and confidence in their abilities (e.g., understanding the material, obtaining good grades in the tests).

Two additional scales were calculated, corresponding to each of the blocks evaluated in the questionnaire (learning strategies and motivation):

- Total learning strategies (12 items). This scale was calculated as the mean of the score of all the items included in the learning strategies block.

- Total motivation (18 items). This scale was calculated as the mean of the score of all the items included in the motivation block.

Table 2

Set of variables considered in the analysis

\begin{tabular}{l|ll}
\hline Variable type & Category & Textual description \\
\hline \multirow{4}{*}{ Dependent } & Learning strategies & Time and study environment \\
& & Critical thinking \\
& & Total learning strategies \\
\cline { 2 - 3 } & \multirow{3}{*}{ Motivation } & Intrinsic goal orientation \\
& & Task value \\
& & Self-efficacy for learning and performance \\
& & Total motivation \\
\hline \multirow{3}{*}{ Independent } & \multirow{3}{*}{ Demographic } & Gender \\
& & Age \\
& & Continent \\
\cline { 2 - 3 } & Prior knowledge & Educational level \\
\cline { 2 - 3 } & MOOC completion & Prior computing knowledge \\
\hline
\end{tabular}

Independent variables

The independent variables considered in this analysis were obtained from the background questions of the survey. The list of independent variables, structured in the order of the research questions, is as follows:

- Gender. Possible self-reported values are male or female.

- Age. Possible self-reported values range from 11 to 100 years old. Other values are not considered feasible and, therefore, entries containing unfeasible values are discarded.

- Continent. This variable is inferred from learners' self-reported country of residence. Possible values are Africa, Asia, Central America and the Caribbean, Europe, North America, South America and Oceania.

- Educational level. This variable is inferred from learners' self-reported highest level of education achieved. Due to the diversity of possible responses, two categories are defined: pre-university (the learner does not claim to have a university degree) and post-university (the learner claims to have a university degree). 
- $\quad$ Prior computing knowledge. This variable indicates if learners self-reported any prior knowledge or experience regarding computing or programming (topics of the MOOCs under study). Possible values are yes and no.

- Percentage of MOOC completion. This variable is calculated through learners' self-reported answers to questions on number of MOOCs enrolled and number of completed MOOCs.

\section{Techniques}

This study aimed to investigate the existence of common characteristics shared by those learners with lower perception of their learning strategies and motivation. To analyse and establish the existence (or not) of relationships among the results of the questionnaire and the background information of the learners, some statistical support was needed. The statistical methods used depended on the type of variables under study (an alpha level of 0.05 was used for all the tests). A dependent variable (quantitative) and an independent variable which is quantitative are analysed using Pearson's correlation coefficient, and an additional graphical plot (to find non-linear relationships visually); these methods were used in RQ1 to analyse the effect of age in learners' SRL skills. A dependent variable (quantitative) and an independent variable which is binary categorical are analysed using T test (particularly unpaired t tests) and Welch's t test (in case the normality assumption is met but the variance homogeneity assumption is violated, as suggested by Ruxton (2006); these methods were used in RQ1 to analyse the effect of gender and educational level in learners' SRL skills, and in RQ2 to analyse the effect of prior computing experience in learners' SRL skills. A dependent variable (quantitative) and an independent variable which is categorical although not binary were analysed using one-way ANOVA between subjects (post-hoc comparisons are done through Tukey-Kramer method to confirm if differences exist, in case sample sizes are unbalanced), and Welch's F test (in case the variance homogeneity assumption is violated, as suggested by Moder (2010), and using Games-Howell for post-hoc comparisons which does not assume variance homogeneity); these methods were used in RQ1 to analyse the effect of continent in learners' SRL skills, and in RQ3 to analyse the effect of MOOC completion percentage in learners' SRL skills.

\section{Educational context}

Three MOOCs on programming (in Java language) throughout several editions were used for survey data collection. Each MOOC lasted 5 weeks, was taught in English and was available in edX. The courses were designed to be taken sequentially, although learners could take them independently, without the need to complete them all. The three consecutive MOOCs together were equivalent to a regular 15-week course. The goal of these MOOCs was to enable people without previous knowledge to learn programming from scratch. The three MOOCs were:

- MOOC1X - Starting to code in Java. Concepts included conditionals, loops, recursive methods and object-oriented programming.

- MOOC2X - Writing Good Code. Concepts included testing, algorithm complexity and refactoring techniques.

- MOOC3X - Fundamental Data Structures and Algorithms. Concepts included data structures such as linked lists, stacks, queues and trees, as well as algorithms applied to them.

Two delivery modes were used in these MOOCs. The first edition of each MOOC always followed an instructor-led mode (weekly release of contents), whereas subsequent editions were released in a self-paced mode (all contents released at the beginning). The collected data corresponds to 4 editions of MOOC1X, 3 editions of MOOC2X and 2 editions of MOOC $3 X$. Altogether, the MOOCs in their multiple editions had almost 409,000 registered learners (see Table 3 for details).

\section{Data collected}

Learners registered in the MOOCs could complete the optional online survey, which was available at the beginning of the course, as part of the induction week. The survey informed the participants that the information collected was to be used for research purposes only. Data was collected anonymously and, therefore, learners could not be mapped with their actual performance in the MOOCs. The data was collected from April 2015 to August 2018. As the three 5-week MOOCs constitute a sequence of contents that results in a regular 15-week course, and as they were offered within a consecutive time frame (which 
makes strong variations in learners' SRL skills unlikely), the data collected from the 3 MOOCs were aggregated to increase the number of entries and give learners several chances to fill in the survey; this led to an initial number of 34,595 entries in total. An entry denotes the data collected after the completion of the survey by one learner. Learner could send their answers after completing the first block or both blocks. As the goal of this study was to find groups whose learning strategies and motivation may have needed more support, only entries of learners who completed the second block were considered. This led to a total of 11,877 valid entries (see Table 3 ).

Table 3

Enrolees and entries from the optional survey on SRL in the three MOOCs

\begin{tabular}{lccc}
\hline MOOC & Enrolees & Total data entries & Valid entries on SRL skills \\
\hline MOOC1X & 340,416 & 33,006 & 11,285 \\
MOOC2X & 45,879 & 1,208 & 460 \\
MOOC 3X & 22,558 & 381 & 132 \\
\hline Total & 408,853 & 34,595 & 11,877 \\
\hline
\end{tabular}

\section{Results}

This section summarises the analysis carried out for each research question and the main findings (Table 4 presents the statistical results). The implications for practice of these results are discussed in the following section.

\section{Differences on self-reported SRL skills among demographic groups (RQ1)}

The literature reports differences of SRL skills according to demographic characteristics. This first part focuses on checking whether these differences applied to the MOOC learners under study or not (RQ1), considering four demographic characteristics: gender, age, continent and educational level.

\section{Gender}

The effect of this qualitative binomial variable (male / female) on the learning strategies and motivation quantitative variables was studied. An independent-samples $t$ test was conducted when comparing gender to time and study environment, critical thinking, total learning strategies, and total motivation. however, variance homogeneity condition was not fulfilled in intrinsic goal orientation, task value, and self-efficacy and, thus, Welch's $t$ test was preferable in these cases.

Regarding learning strategies, there was a significant difference in total learning strategies and critical thinking. In the first case, men $(N=9110, M=4.68)$ scored lower than women $(N=2767, M=4.77)$, $t(11875)=-4.54, p<.001$, whereas the situation was the opposite in the second case, between men $(M=$ $5.23)$ and women $(M=5.07), t(11875)=7.1, p<.001$. Therefore, in the MOOCs under study, and considering that data was self-reported, women reported to be able to organise their time and study place better, whereas men reported to be able to perform more critical evaluations to the new presented concepts or ideas. however, as the mean of women was higher in time and study environment while men stood out in critical thinking scale, their scores were balanced in the total learning strategies variable and, thus, no significant difference was found in the total mean of learning strategies.

Regarding motivation, significant differences were found in task value $[t(4753)=-2.81, p=.005]$ and selfefficacy $[t(4322)=8.49, p<.001]$, whereas intrinsic goal orientation was similar in both groups. Men reported higher self-efficacy than women $(M=5.75$ and $M=5.55$ respectively) but did not consider the material as useful as women did, since their task value score was lower $(M=6.08$ and $M=6.14$ respectively). In general (total motivation), men $(M=5.89)$ had higher motivation than women $(M=5.82)$, $t(11875)=3.73, p<.001$.

Age

The effect of this quantitative variable was studied. As the dependent variables were also quantitative, the possible relationship was analysed through statistical correlation. Pearson's coefficient was calculated with all dependent variables, but all relationships were weak and negligible $(|r|<0.11)$. To find non-linear relationships (e.g., quadratic, cubic), plots were used, although no dependence between the variables was 
observed. Even though age could be expected to have a significant influence on learners' SRL skills (Kizilcec et al., 2017), its effect could not be verified in this analysis (always taking into account that data was self-reported). This result seemed to indicate that age was not a determining factor in learners' SRL skills because people of the same age but with different backgrounds, interests and cultures could possess very diverse skills. Therefore, age alone was not a decisive factor in the learners registered in the MOOCs under analysis but could be influential when combined with other variables (e.g., country, education level).

\section{Continent}

The effect of geographical location was studied. Countries of residence of the learners were classified in 7 geographical areas. However, due to the considerable gap among sample sizes in each group, only the three most popular areas were analysed: Asia, 4157 entries; North America, 2496 entries; and Europe, 2466 entries.

The statistical comparison was performed through a one-way ANOVA between subjects or Welch's $F$ test, depending on the homogeneity of variance of the data (Levene's test was used to check this assumption). In all the 7 compared scales, significant differences were found. After the post-hoc comparisons, differences among all the groups were identified for scales time and study environment, total learning strategies, intrinsic goal orientation, self-efficacy, and total motivation. In critical thinking, significant differences were found between Europe and the other 2 groups $[F(2,5451)=41, p<.001]$. In both cases, learners from Europe scored 0.22 less compared to learners from North America and Asia $(p<.001)$. In task value, North America stood out from the other two areas $[F(2,5566)=43.7, p<.001]$. Games-Howell showed a difference between learners from North American and learners from Asia of 0.19 points $(p<.001)$, and between the former and learners from Europe of $0.2(p<.001)$.

North America showed higher scores in all the scales under analysis. Learners from North America perceived that they had better SRL skills than learners from the other two areas, whereas Europeans ranked the lowest in all the scales except for time and study environment. However, the difference between learners from Europe and Asia was just of 0.07 points $(p=.0053)$. Therefore, the geographical location had some effect on the skills of the learners in the MOOCs under study, which might be due to cultural impact either on the skills or on the perception of abilities. It is also worth mentioning that the joint effect of the independent variables continent and educational level was studied and the proportion of pre-university and post-university learners was similar per continent, so the differences in relation to SRL skills between North America and Europe cannot be attributed to variations in the self-reported educational levels of MOOC learners. Although these findings considered broad geographical areas (continents), these differences might be influenced more by some countries. Specifically, most of the North American entries corresponded to the United States of America (72\%), and most of the Asian entries corresponded to India (63\%). In Europe, the samples were more distributed among several countries.

\section{Educational level}

The effect of a pre- or post-university stage was studied. Learners were divided in these two groups $(N=$ 4885 and $N=6800$ respectively) and analysed by means of unpaired students' $t$ test (and Welch's $t$ test when homogeneity variance assumption was not met). It is worth mentioning that the educational level referred to the highest completed level. Thus, university students were considered as pre-university learners until they obtain a bachelor's degree.

Regarding learning strategies, significant differences were found in all the scales. For example, in critical thinking, post-university learners scored 0.12 points higher than pre-university learners $[t(10231)=-6, p<$ $.001]$. In general (total learning strategies), significant differences were found between post-university and pre-university learners $[t(10265)=-6.18, p<.001]$. However, this difference was only 0.1 points as the influence of time and study environment (with 7 items) was higher than that of critical thinking (with 5 items) when calculating the mean of the learning strategies scale.

Regarding motivation, significant differences were found in task value $[t(11683)=3.1, p=.002]$ and selfefficacy $[t(10278)=-4.32, p<.001]$, but not in intrinsic goal orientation. Pre-university learners scored better in task value than post-university learners, with estimated means of 6.13 and 6.07 points respectively. Thus, the formers seemed to perceive that the course was more interesting and important. However, findings suggested that the university stage helped post-university learners develop self-efficacy $(M=5.74)$ 
compared to pre-university learners $(M=5.65)$. Moreover, no significant differences were found between the educational level and the total motivation scale $[t(11683)=-1.42, p=0.16]$.

\section{Differences on self-perceived motivation and learning strategies according to prior computing experience (RQ2)}

The second part focused on analysing other abilities which could be useful in the context of MOOCs on programming (RQ2). Learners could have perceived the quality of their SRL skills towards the MOOCs differently depending on how familiar they were with the learning context. For example, knowing about other programming languages could have motivated learners or increased their confidence in completing the MOOC under study. The experience with computing or programming was the binomial categorical variable whose effect on the self-reported skills was studied. Students' $t$ test was applied when the dependent variable was total learning strategies as the normality and variance homogeneity assumptions were met. In the other cases, heteroscedasticity was observed, and Welch's $t$ tests were applied.

The analysis did not find significant differences on the time and study environment of experienced $(N=$ $7861)$ and unexperienced $(N=4016)$ learners $[t(11875)=-0.56, p=.58]$. This suggested that being familiar with computing or programming did not necessarily relate to how learners organised their time or chose their study place. In total learning strategies, significant differences were identified, especially in critical thinking $[t(7725)=-7.43, p<.001]$, as experienced learners scored 0.16 points higher in this skill than unexperienced ones $(M=5.09$ and $M=5.25$ respectively). It was found that previous experience could contribute to critical thinking, as learners were able to apply their previous knowledge to evaluate new concepts or make critical evaluations.

The analysis found significant differences on learners' self-reported motivation, mainly on self-efficacy $[t(7302)=-14.42, p<.001]$, as the score of experienced learners $(M=5.81)$ was 0.31 higher. In total motivation, this difference was reduced to 0.2 points $[t(7438)=-10.79, p<.001]$, with an estimated mean of 5.94 points. These findings supported the idea that learners who were more familiar with the context could feel more confident towards his/her success and skills, and, thus, were more motivated to succeed in the MOOC.

\section{Differences on learners' SRL skills according to previous MOOC completion percentage (RQ3)}

The last part of this analysis was focused on the possible effect that completing other MOOCs (RQ3) could have in the improvement of SRL skills. For this analysis, the percentage of completed MOOCs divided by the total MOOCs in which the learner registered was calculated. Three groups were created, according to the percentage obtained:

- Low completers (completion range 0\%-20\%)

- Medium completers (completion range 20\%-60\%)

- High completers (completion range 60\%-100\%).

This analysis involved one quantitative dependent variable and one categorical variable with 3 groups. Sample sizes were $N=5648, N=2410$, and $N=3759$ for low, medium and high completers respectively. One-way ANOVA between subjects was applied in total learning strategies, and Welch's $F$ test in the other cases, as the variance homogeneity assumption was violated.

Except for the critical thinking scale in which Games-Howell post-hoc showed significant differences among all the groups (all $p<.008$ ), significant differences were observed in the rest of scales between high completers and the other two groups (see Table 4). In total learning strategies $[F(2,6243)=20.9, p<.001]$, high completers scored 0.26 points higher than low completers $(p<.001)$ and 0.25 higher than medium completers $(p<.001)$. In total motivation $[F(2,6243)=20.9, p<.001]$, high completers scored 0.12 points higher than low completers $(p<.001)$ and 0.1 lower than medium completers $(p<.001)$. All the analyses showed that high completers scored the highest among the groups, while low completers showed poor learning strategies and motivation. 
Table 4

Summary of significant results

\begin{tabular}{|c|c|c|c|c|c|c|c|c|}
\hline \multirow[t]{2}{*}{$\overline{\mathrm{RQ}}$} & & \multicolumn{3}{|c|}{ Learning strategies } & \multicolumn{4}{|c|}{ Motivation } \\
\hline & Variables & T \& $S$ env. & CT & LS mean & IGO & Task value & SE for $L \& P$ & $\begin{array}{l}\text { Motivation } \\
\text { mean }\end{array}$ \\
\hline \multirow{9}{*}{1} & Gender & $t=-4.54$ & $t=7.1$ & - & - & $t w=-2.81$ & $t w=8.49$ & $t=3.73$ \\
\hline & $\mathrm{M}-\mathrm{F}$ & $-.093 * * *$ & $.168 * * *$ & & & $.056 * *$ & $.204 * * *$ & $.074 * * *$ \\
\hline & Age & $r=.103 * * *$ & $r=.02 *$ & $r=.08 * * *$ & - & $r=-.024 *$ & - & - \\
\hline & Cont. & $F w=39.61 * * *$ & $F w=40.99 * * *$ & $F w=33.42 * * *$ & $F w=21.4 * * *$ & $F w=43.78$ & $F=41.71 * * *$ & $F w=41.39 * * *$ \\
\hline & EU-AS & $.072 * *$ & $-.217 * * *$ & $-.048 *$ & $-.069 *$ & - & $-.193 * * *$ & $-.103 * * *$ \\
\hline & NA-AS & $.218^{* * *}$ & - & $.132 * * *$ & $.096^{* * *}$ & $.194 * * *$ & $.069 *$ & $.117 * * *$ \\
\hline & NA-EU & $.146^{* * *}$ & $.229 * * *$ & $.181 * * *$ & $.165^{* * *}$ & $.201 * * *$ & $.262 * * *$ & $.22 * * *$ \\
\hline & Ed. level & $t w=-4.23$ & $t w=-6$ & $t w=-6.18$ & - & $t=3.1$ & $t w=-4.32$ & - \\
\hline & Pre-Post & $-.076^{* * * *}$ & $-.124 * * *$ & $-.095 * * *$ & & $.054 * *$ & $.087 * * *$ & \\
\hline 2 & $\begin{array}{l}\mathbf{C E} \\
\text { No prior- } \\
\text { Prior }\end{array}$ & - & $\begin{array}{l}t w=-7.43 \\
-.16^{*} * *\end{array}$ & $\begin{array}{l}t w=-4.52 \\
-.073 * * *\end{array}$ & $\begin{array}{l}t w=-6.58 \\
-.13 * * *\end{array}$ & $\begin{array}{l}t w=-5.28 \\
-.099 * * *\end{array}$ & $\begin{array}{l}t w=-14.42 \\
-.307 * * *\end{array}$ & $\begin{array}{l}t w=-10.79 \\
-.197 * * *\end{array}$ \\
\hline \multirow{4}{*}{3} & $\begin{array}{l}\text { MOOC } \\
\text { comp. } \\
(\%)\end{array}$ & $F=135.6^{* * *}$ & $F w=47.94 * * *$ & $F w=135.52 * * *$ & $F w=18.41 * * *$ & $F w=7.28 * * *$ & $F w=28.11^{* * *} *$ & $F w=20.91 * * *$ \\
\hline & $\mathrm{MC}-\mathrm{LC}$ & - & $.08 * *$ & - & - & - & - & - \\
\hline & $\mathrm{HC}-\mathrm{LC}$ & $.292 * * *$ & $.219 * * *$ & $.222 * * *$ & $.119 * * *$ & $.056^{*}$ & $.11 * * *$ & $.117 * * *$ \\
\hline & HC-MC & $.271 * * *$ & $.139 * * *$ & $.201 * * *$ & $.082 * *$ & $.083 * *$ & $.058 * * *$ & $.1 * * *$ \\
\hline
\end{tabular}

Note. - not significant $(\alpha=.05) ; *$ significant at $p<.05 ; * *$ significant at $p<.01 ; * * *$ significant at $p<.001$

$\mathrm{T} \& \mathrm{~S}$ env. = Time and study environment; CT $=$ Critical thinking; LS = Learning strategies; IGO = Intrinsic goal orientation; SE for L \& P = Self-efficacy for learning and performance; Cont. = Continent; EU = Europe; AS = Asia; NA = North America; Ed. level = Educational level; Pre-Post = Pre-university, Post-university; CE = Computing experience; $\mathrm{LC}=$ low completers; $\mathrm{MC}=$ medium completers; $\mathrm{HC}=$ high completers.

$t=$ Student's $t$ - test; $t w=$ Welch's $t$ test; $F=$ one-way ANOVA; $F w=$ Welch's $F$ test.

\section{Implications for practice}

This study served to gain insights on SRL strategies and motivation of MOOC learners using self-reported data collected from a series of three MOOCs on programming. Some groups that may be at greater risk of dropout due to having low motivation and/or poor SRL strategies were identified based on their characteristics. Instructors can personalise some aspects of the MOOCs with the aim to target the identified groups with respect to the following characteristics.

\section{Gender}

Gender differences in self-reported learning strategies were not found to be statistically significant when considering learning strategies in general. This is consistent with the research study by Liou and Kuo (2014). However, differences were found when considering particular learning strategies, as also found by Kizilcec et al. (2017). Men reported higher critical thinking skills, and women reported better time and study environment skills. This latter finding is also consistent with the work by Duckworth and Seligman (2006), which found that women are more self-disciplined. Instructors may create gender cohorts and offer some tips to the male cohort about managing time and the study environment and include some additional discussions for the female cohort to promote critical thinking on the course subject. In any case, more research is needed comparing self-reported learners' perception and their observed behaviour. Regarding motivation, men reported being more motivated when starting the MOOCs. This result could be influenced by the topic of the MOOCs, in this case, computer programming. Stereotypes would suggest that programming is a topic in which men are usually more interested than women (Cheryan et al., 2017). In fact, the proportion of male learners is usually much higher in MOOCs on computer science, as it also happened in the MOOCs under analysis. Instructors could send specific messages to the female cohort explaining the role of women in the development of computer science.

\section{Age}

No significant differences were found in relation to the age of the learners, despite the initial hypothesis that being older could mean a higher level of SRL skills to undertake the MOOCs. This is the opposite of the findings by Guo and Reinecke (2014), who concluded that the navigation strategies of MOOC learners 
differ with age. The age differences between participants can be exploited to foster discussions on the development of computing in the last decades by contrasting personal experiences (e.g., describing how their first computer and operating system were, how they approached the world of computing or what the first programming language they learned was). All in all, new research studies should delve deeper in the role of age, together with other demographic factors (such as the educational level or the country of origin), in the development of SRL skills by MOOC learners.

\section{Continent}

Europeans reported poorer learning strategies and lower motivation than learners from Asia and North America. Therefore, instructors should pay more attention to Europeans as these could be considered a group at risk, who could need additional guidance in the MOOCs under study. Instructors could create cohorts by country of origin (or continent) and offer some tips to European learners on time management, study environment management and critical thinking. Likewise, strategies could be implemented for this cohort to gain confidence in an induction week in the MOOC. All in all, findings suggest that cultural differences affected the perception of skills, although new research studies should compare self-reported learners' perception and their observed behaviour to see if learners tend to overestimate or underestimate their SRL skills depending on the region from which they come from.

\section{Educational level}

Learners with university experience reported higher SRL skills than learners without university experience. This is aligned with the findings by Hood et al. (2015), who stated that learners who have studied at university have better learning strategies than learners who did not achieve that educational level. Therefore, instructors should pay more attention to pre-university learners, who could be considered a group at risk of dropping out the MOOCs. To improve the learning strategies of pre-university learners, instructors could, for instance, create a cohort with pre-university learners and provide some extra material to this cohort. This extra material could include a code of good practices in online learning environments, with the aim of teaching these learners how to organise their study time or to set up a good study environment. In addition, instructors could encourage learners in this pre-university cohort to set goals before completing their tasks and reflect on their performance at the end of each module or week of the MOOC. These actions target the three phases in SRL: preparatory, performance and appraisal. Interestingly, the university stage did not appear to encourage and intrinsically motivate learners differently compared to pre-university learners. Reasons for registering in these MOOCs on programming could be connected more to the extrinsic motivation of the learners. Some learners might have registered because the topic was appealing for them and they were interested in programming (and thus had high motivation), whereas others might need to learn about programming to advance in their studies or career.

\section{Computing experience}

The familiarity level with the context of the MOOCs influenced learners' perception of both their learning strategies and motivation. This finding aligns with other research studies, such as the one by Littlejohn et al. (2016) or by Milligan et al. (2016), who found that higher self-efficacy was associated with previous experience with related MOOCs. For a learner without experience in computing, enrolling in the MOOCs under analysis may represent an important challenge (due to the delivery format and the course syllabus). Therefore, instructors should pay more attention to learners without experience in online education in general and without or little prior experience on the MOOC topic in particular. For example, in the MOOCs under study, it might be interesting to create a cohort for learners without prior computing experience and provide them with extra content on the fundamentals of computing to better contextualise the basis for the MOOCs.

\section{MOOC completion}

A significant relationship was found between the SRL skills of the learners and the percentage of MOOCs they reported to have completed before joining any of the MOOCs in the series. Results suggested that learners who had completed only a small percentage of MOOCs could find more difficulties. Therefore, instructors should pay more attention to low completers as this might be an important high-risk group. For example, a specific cohort could be created for low completers with an extensive induction module 
explaining how the content is organised in the MOOC platform and the main issues that typically lead to learners' dropout in MOOCs (Eriksson et al., 2017). Nonetheless, this significant relationship should be further studied, to find the directionality. Are learners improving their SRL skills by completing MOOCs? Or are the high self-regulated learners the only ones who can complete MOOCs (as the skills do not change)? Further analysis on this question is encouraged, for example, by means of non-anonymous preand post-MOOC surveys.

\section{Conclusions}

This study identified several groups at risk of dropping out of a series of MOOCs on programming based on a self-reported questionnaire, with the first part designed to obtain learners' demographic characteristics and the second part to collect self-reported learners' SRL skills. It is important to keep in mind that there is a very close relationship between SRL skills and motivation. Learners may have high SRL skills at applying learning strategies but choose not to do so due to lack of motivation to learn the specific content of a MOOC. As a general summary, and for the MOOCs under study, which belong to the area of computer science, higher risk groups were found to be Europeans, learners without any experience with computing or programming and learners who had completed half or fewer of the MOOCs in which they registered. Moreover, women might need extra motivation and men tips on time management and study environment management. Finally, pre-university learners might need specific guidance to improve their learning strategies. Specific cohorts based on demographic data could be created in the MOOC to address these groups. Finally, it is important to keep in mind that when people do not self-regulate their learning in MOOCs, it does not necessarily mean that they do not possess self-regulation skills. That person may choose not to engage fully in the MOOC (as these courses can be used flexibly without penalty and at no cost), and simply explore the content of the course.

The limitations of this study make it difficult to generalise results in other educational contexts, and further research in this area is needed to support or discard the findings obtained here. These limitations include the self-reported nature of the survey used to collect data, where answers relied on the accuracy of learners to evaluate their SRL skills. However, self-reported instruments are considered to be a suitable approach as long as their results are understood to be relative to the particular context and individuals (Fontana et al., 2015). To have further support, the survey could be modified so as not to be anonymous. This modification would enable future research studies to compare the answers of self-reported learning strategies and the real behaviour of the learners. In addition, we are aware that a learner who registered in more than one MOOC of the series could have decided to answer the survey more than once, which could bias the results. However, due to the optionality of completing the survey, it is unlikely that a learner would have decided to complete the same survey twice. What is more, the answers came mostly from learners from the first MOOC, which was consistent with the number of registrations as the first MOOC comprised most of the registered learners from the three MOOCs. Finally, the questionnaire used to collect data comprises only a subset of the MSLQ, since the questionnaire, initially designed for a classroom environment, had to be adapted to the MOOC context. In the future, more scales or items from the MSLQ may be added or other questionnaires that better fit the MOOC context could be used. Finally, an inherent challenge to research studies on MOOCs is obtaining absolute conclusions. This analysis was applied to a set of three programming MOOCs on the edX platform and, therefore, results are not necessarily generalis able to other contexts or settings that differ substantially. In those cases, researchers are encouraged to develop similar analyses to examine the generalisability of this study.

All in all, future research directions should focus not only on understanding and measuring the SRL skills of MOOC learners but also on developing interventions to help develop those SRL skills. In today's world, with the COVID-19 pandemic, educational institutions had to move classes online overnight, but learners have not received any training in relation to the development of their SRL skills. While MOOCs have increased interest in SRL skills in online learning, mainly due to the lack of teacher support in these massive environments and the high dropout rates, the widespread adoption of online learning worldwide should serve to put a stronger emphasis on research related to the study and improvement of SRL at all educational levels; otherwise, many learners may be left behind because they lack the required SRL skills. 


\section{Acknowledgements}

This work was supported in part by the FEDER/Ministerio de Ciencia, Innovación y UniversidadesAgencia Estatal de Investigación, through the Smartlet Project under Grant TIN2017-85179-C3-1-R, and in part by the Madrid Regional Government through the e-Madrid-CM Project under Grant S2018/TCS4307, a project which is co-funded by the European Structural Funds (FSE and FEDER). Partial support has also been received from the European Commission through Erasmus + Capacity Building in the Field of Higher Education projects, more specifically through projects LALA, InnovaT and PROF-XXI (586120EPP-1-2017-1-ES-EPPKA2-CBHE-JP), (598758-EPP-1-2018-1-AT-EPPKA2-CBHE-JP), (609767-EPP1-2019-1-ES-EPPKA2-CBHE-JP). This publication reflects the views only of the authors, and funders cannot be held responsible for any use which may be made of the information contained therein.

\section{References}

Alario-Hoyos, C., Estévez-Ayres, I., Pérez-Sanagustín, M., Delgado Kloos, C., \& Fernández-Panadero, C. (2017). Understanding learners' motivation and learning strategies in MOOCs. The International Review of Research in Open and Distributed Learning, 18(3), 119-137. https://doi.org/10.19173/irrodl.v18i3.2996

Alonso-Mencía, M. E., Alario-Hoyos, C., Maldonado-Mahauad, J., Estévez-Ayres, I., Pérez-Sanagustín, M., \& Delgado Kloos, C. (2020). Self-regulated learning in MOOCs: Lessons learned from a literature review. Educational Review, 72(3), 319-345. https://doi.org/10.1080/00131911.2019.1566208

Barnard-Brak, L., Paton, V.O., \& Lan, W. Y.(2010). Profiles in self-regulated learning in the online learning environment. The International Review of Research in Open and Distributed Learning, 11(1), 61-80. https://doi.org/10.19173/irrodl.v11i1.769

Bozkurt, A., \& Akbulut, Y. (2019). Dropout patterns and cultural context in online networked learning spaces. Open Praxis, 11(1), 41-54. https://doi.org/10.5944/openpraxis.11.1.940

Campbell, J., Gibbs, A. L., Najafi, H., \& Severinski, C. (2014). A comparison of learner intent and behaviour in live and archived MOOCs. The International Review of Research in Open and Distributed Learning, 15(5). https://doi.org/10.19173/irrodl.v15i5.1854

Chen, Y.-H., \& Chen, P.-J. (2015). MOOC study group: Facilitation strategies, influential factors, and student perceived gains. Computers \& Education, 86, 55-70. https://doi.org/10.1016/j.compedu.2015.03.008

Cheryan, S., Ziegler, S. A., Montoya, A. K., \& Jiang, L. (2017). Why are some STEM fields more gender balanced than others? Psychological Bulletin, 143(1), 1-35. https://doi.org/10.1037/bul0000052

Clow, D. (2013). MOOCs and the funnel of participation. In D. Suthers, K. Verbert, E. Duval, \& Z. Ochoa (Eds.), Proceedings of the 3rd International Conference on Learning Analytics and Knowledge (pp. 185-189). Association for Computing Machinery. https://doi.org/10.1145/2460296.2460332

Creswell, J. W. (2002). Educational research: Planning, conducting, and evaluating. Merrill Prentice Hall.

Davis, D., Jivet, I., Kizilcec, R. F., Chen, G., Hauff, C., \& Houben, G.-J. (2017). Follow the successful crowd: Raising MOOC completion rates through social comparison at scale. In A. Wise, P. H. Winne, \& G. Lynch (Eds.), Proceedings of the 7th International Conference on Learning Analytics and Knowledge (pp. 454-463). Association for Computing Machinery. https://doi.org/10.1145/3027385.3027411

De Barba, P., Kennedy, G. E., \& Ainley, M. (2016). The role of students' motivation and participation in predicting performance in a MOOC. Journal of Computer Assisted Learning, 32(3), 218-231. https://doi.org/10.1111/jcal.12130

Duckworth, A. L., \& Seligman, M. E. (2006). Self-discipline gives girls the edge: Gender in selfdiscipline, grades, and achievement test scores. Journal of Educational Psychology, 98(1), 198-208. https://doi.org/10.1037/0022-0663.98.1.198

Eriksson, T., Adawi, T., \& Stöhr, C. (2017). Time is the bottleneck: A qualitative study exploring why learners drop out of MOOCs. Journal of Computing in Higher Education, 29(1), 133-146. https://doi.org/10.1007/s12528-016-9127-8

Fontana, R. P., Milligan, C., Littlejohn, A., \& Margaryan, A. (2015). Measuring self-regulated learning in the workplace. International Journal of Training and Development, 19(1), 32-52.

https://doi.org/10.1111/ijtd.12046 
Gašević, D., Kovanović, V., Joksimović, S., \& Siemens, G. (2014). Where is research on massive open online courses headed? A data analysis of the MOOC Research Initiative. The International Review of Research in Open and Distributed Learning, 15(5). https://doi.org/10.19173/irrodl.v15i5.1954

Guo, P. J., \& Reinecke, K. (2014). Demographic differences in how students navigate through MOOCs. In M. Sahami, A. Fox, M A. Hearst, \& M. T. H. Chi (Eds), Proceedings of the 1st ACM Conference on Learning@ Scale Conference (pp. 21-30). Association for Computing Machinery. https://doi.org/10.1145/2556325.2566247

Halawa, S., Greene, D., \& Mitchell, J. (2014). Dropout prediction in MOOCs using learner activity features. In U. Cress \& C. Delgado Kloos (Eds.), Proceedings of the 2nd European MOOC Stakeholder Summit (pp. 58-65). Open Education Europa.

Hood, N., Littlejohn, A., \& Milligan, C. (2015). Context counts: How learners' contexts influence learning in a MOOC. Computers \& Education, 91, 83-91. https://doi.org/10.1016/j.compedu.2015.10.019

Jo, Y., Tomar, G., Ferschke, O., Rosé, C. P., \& Gasevic, D. (2016). Expediting support for social learning with behavior modeling. In T. Barnes, M. Chi, \& M. Feng (Eds.), Proceedings of the 9th International Conference on Educational Data Mining (pp. 400-405). The International Educational Data Mining Society. https://www.educationaldatamining.org/EDM2016/proceedings/edm2016 shortpapers.pdf

Khalil, H., \& Ebner, M. (2014). MOOCs completion rates and possible methods to improve retention: A literature review. In J. Herrington, J. Viteli, \& M. Leikomaa (Eds.), Proceedings of EdMedia 2014: World Conference on Educational Media and Technology (pp. 1305-1313). Association for the Advancement of Computing in Education. https://www.learntechlib.org/primary/p/147656/

Kizilcec, R. F., Pérez-Sanagustín, M., \& Maldonado, J. J. (2016). Recommending self-regulated learning strategies does not improve performance in a MOOC. In J. Haywood, V. Aleven, J. Kay, \& I. Roll (Eds.), Proceedings of the 3rd ACM Conference on Learning@ Scale (pp. 101-104). Association for Computing Machinery. https://doi.org/10.1145/2876034.2893378

Kizilcec, R. F., Pérez-Sanagustín, M., \& Maldonado, J. J. (2017). Self-regulated learning strategies predict learner behavior and goal attainment in massive open online courses. Computers \& Education, 104, 18-33. https://doi.org/10.1016/j.compedu.2016.10.001

Kocdar, S., Karadeniz, A., Bozkurt, A., \& Buyuk, K. (2018). Measuring self-regulation in self-paced open and distance learning environments. International Review of Research in Open and Distributed Learning, 19(1), 25-43. https://doi.org/10.19173/irrodl.v19i1.3255

Kornell, N., \& Finn, B. (2016). Self-regulated learning: An overview of theory and data. In J. Dunlosky \& S. K. Tauber (Eds.), The Oxford handbook of metamemory (pp. 325-340). Oxford.

Lee, D., Watson, S. L., \& Watson, W. R. (2019). Systematic literature review on self-regulated learning in massive open online courses. Australasian Journal of Educational Technology, 35(1), 28-41. https://doi.org/10.14742/ajet.3749

Liou, P.-Y., \& Kuo, P.-J. (2014). Validation of an instrument to measure students' motivation and selfregulation towards technology learning. Research in Science \& Technological Education, 32(2), 79 96. https://doi.org/10.1080/02635143.2014.893235

Littlejohn, A., Hood, N., Milligan, C., \& Mustain, P.(2016). Learning in MOOCs: Motivations and selfregulated learning in MOOCs. The Internet and Higher Education, 29, 40-48. https://doi.org/10.1016/j.iheduc.2015.12.003

Maldonado-Mahauad, J., Pérez-Sanagustín, M., Kizilcec, R. F., Morales, N., \& Munoz-Gama, J. (2018). Mining theory-based patterns from big data: Identifying self- regulated learning strategies in massive open online courses. Computers in Human Behavior, 80, 179-196. https://doi.org/10.1016/j.chb.2017.11.011

Milligan, C., Littlejohn, A., \& Hood, N. (2016). Learning in MOOCs: A comparison study. In M. Khalil, M. Ebner, M. Kopp, A. Lorenz, \& M. Kalz (Eds.), Proceedings of the European Stakeholder Summit on Experiences and Best Practices in and around MOOCs (pp. 15-26). Books on Demand.

Moder, K. (2010). Alternatives to $F$-test in one way ANOVA in case of heterogeneity of variances (a simulation study). Psychological Test and Assessment Modeling, 52(4), 343-353. http://www.psychologie-aktuell.com/fileadmin/download/ptam/4-2010 20101218/01 Moder.pdf

Onah, D. F., Sinclair, J., \& Boyatt, R. (2014). Dropout rates of massive open online courses: Behavioural patterns. In L. Gómez Chova, A. López Martínez, \& I. Candel Torres (Eds.), Proceedings of the 6th International Conference on Education and New Learning Technologies (pp. 5825-5834). IATED. https://doi.org/10.13140/RG.2.1.2402.0009

Panadero, E. (2017). A review of self-regulated learning: Six models and four directions for research. Frontiers in Psychology, 8. https://doi.org/10.3389/fpsyg.2017.00422 
Pérez-Álvarez, R., Pérez-Sanagustín, M., \& Maldonado-Mahauad, J. J. (2017). NoteMyProgress: Supporting learners' self-regulated strategies in MOOCs. In E. Lavoué, H. Drachsler, K. Verbert, J. Broisin, \& M. Pérez-Sanagustín (Eds.), European Conference on Technology Enhanced Learning (pp. 517-520). Springer. https://doi.org/10.1007/978-3-319-66610-5 43

Phan, T., McNeil, S. G., \& Robin, B. R. (2016). Students' patterns of engagement and course performance in a massive open online course. Computers \& Education, 95, 36-44. https://doi.org/10.1016/j.compedu.2015.11.015

Pintrich, P. R. (2000a). An achievement goal theory perspective on issues in motivation terminology, theory, and research. Contemporary Educational Psychology, 25(1), 92-104. https://doi.org/10.1006/ceps.1999.1017

Pintrich, P. R. (2000b). The role of goal orientation in self-regulated learning. In M. Boekaerts, P. R. Pintrich, \& M. Zeidner (Eds.), Handbook of self-regulation (pp. 451-502). Elsevier. https://doi.org/10.1016/B978-012109890-2/50043-3

Pintrich, P., Smith, D, Garcia, T, \& McKeachie, W. (1991). A manual for the use of the motivated strategies for learning questionnaire (Technical Report 91-b-004). The Regents of the University of Michigan.

Puustinen, M., \& Pulkkinen, L. (2001). Models of self-regulated learning: A review. Scandinavian Journal of Educational Research, 45(3), 269-286. https://doi.org/10.1080/00313830120074206

Ruxton, G. D. (2006). The unequal variance t-test is an underused alternative to Student's t-test and the Mann-Whitney U test. Behavioral Ecology, 17(4), 688-690. https://doi.org/10.1093/beheco/ark016

Watson, S. L., Watson, W. R., Yu, J. H., Alamri, H., \& Mueller, C. (2017). Learner profiles of attitudinal learning in a MOOC: An explanatory sequential mixed methods study. Computers \& Education, 114, 274-285. https://doi.org/10.1016/j.compedu.2017.07.005

Zimmerman, B. J. (2000). Attaining self-regulation: A social cognitive perspective. In M. Boekaerts, P. R. Pintrich, \& M. Zeidner (Eds.), Handbook of self-regulation (pp. 13-39). Elsevier. https://doi.org/10.1016/B978-012109890-2/50031-7

Zimmerman, B. J. (2002). Becoming a self-regulated learner: An overview. Theory into Practice, 41(2), 64-70. https://doi.org/10.1207/s15430421tip4102_2

Zimmerman, B. J., \& Pons, M. M. (1986). Development of a structured interview for assessing student use of self-regulated learning strategies. American Educational Research Journal, 23(4), 614-628. https://doi.org/10.3102\%2F00028312023004614

Corresponding author: Carlos Alario-Hoyos, calario@it.uc3m.es

Copyright: Articles published in the Australasian Journal of Educational Technology (AJET) are available under Creative Commons Attribution Non-Commercial No Derivatives Licence (CC BY-NC-ND 4.0). Authors retain copyright in their work and grant AJET right of first publication under CC BY-NC-ND 4.0.

Please cite as: Alonso-Mencía, M. E., Alario-Hoyos, C., Estévez-Ayres, I., \& Delgado Kloos, C. (2021). Analysing self-regulated learning strategies of MOOC learners through self-reported data. Australasian Journal of Educational Technology, 37(3), 56-70. https://doi.org/10.14742/ajet.6150 


\section{Appendix}

The appendix summarises the subset of MSLQ items that comprised the survey, organised by scale and question number in the survey.

\begin{tabular}{|c|c|c|}
\hline \multicolumn{3}{|r|}{ Learning strategies } \\
\hline \multirow{7}{*}{$\begin{array}{l}\text { Time and study } \\
\text { environment }\end{array}$} & 1. & I usually study in a place where I can concentrate on my course work. \\
\hline & 3. & I make good use of my study time. \\
\hline & 6. & I find it hard to stick to a study schedule. \\
\hline & 7. & I have a regular place set aside for studying. \\
\hline & 9. & $\begin{array}{l}\text { I will make sure that I keep up with the weekly videos and assignments for } \\
\text { this course. }\end{array}$ \\
\hline & 11. & $\begin{array}{l}\text { I often find that I don't spend very much time on online course that I've } \\
\text { taken because of other activities. }\end{array}$ \\
\hline & 12. & I rarely find time to review my notes before an exam. \\
\hline \multirow[t]{5}{*}{ Critical thinking } & 2. & $\begin{array}{l}\text { I often find myself questioning things I hear or read in course that I am } \\
\text { taking to decide if I find them convincing. }\end{array}$ \\
\hline & 4. & $\begin{array}{l}\text { When a theory, interpretation, or conclusion is presented in a course, I try } \\
\text { to decide if there is good supporting evidence. }\end{array}$ \\
\hline & 5. & $\begin{array}{l}\text { When I study, I treat the course material as a starting point and try to } \\
\text { develop my own ideas about it. }\end{array}$ \\
\hline & 8. & I try to play around with ideas of my own related to what I am learning. \\
\hline & 10 . & $\begin{array}{l}\text { Whenever I read or hear an assertion or conclusion in a course, I think about } \\
\text { possible alternatives. }\end{array}$ \\
\hline \multicolumn{3}{|r|}{ Motivation } \\
\hline \multirow[t]{4}{*}{$\begin{array}{l}\text { Intrinsic goal } \\
\text { orientation }\end{array}$} & 13. & $\begin{array}{l}\text { In a course like this, I prefer course material that really challenges me, so I } \\
\text { can learn new things. }\end{array}$ \\
\hline & 20. & $\begin{array}{l}\text { In a course like this, I prefer course material that arouses my curiosity, even } \\
\text { if it is difficult to learn. }\end{array}$ \\
\hline & 24. & $\begin{array}{l}\text { The most satisfying thing for me in this course will be trying to understand } \\
\text { the content as thoroughly as possible. }\end{array}$ \\
\hline & 26. & $\begin{array}{l}\text { When I have the opportunity in this kind of courses, I try to do all the } \\
\text { exercises and course assignments that I can learn from even if they don't } \\
\text { guarantee a good grade. }\end{array}$ \\
\hline \multirow[t]{6}{*}{ Task value } & 14. & I think I will be able to use what I will learn in this course in other courses. \\
\hline & 17. & It is important for me to learn the material in this course. \\
\hline & 21. & I am very interested in the content area of this course. \\
\hline & 25 . & I think the course material in this course will be useful for me to learn. \\
\hline & 27. & I like the subject matter of this course. \\
\hline & 28. & Understanding the subject matter of this course is very important to me. \\
\hline \multirow{8}{*}{$\begin{array}{l}\text { Self-efficacy for } \\
\text { learning and } \\
\text { performance }\end{array}$} & 15. & I believe I will receive an excellent grade in this course. \\
\hline & 16. & $\begin{array}{l}\text { I am certain I can understand the most difficult material presented in this } \\
\text { course. }\end{array}$ \\
\hline & 18. & I am confident I can learn the basic concepts taught in this course. \\
\hline & & $\begin{array}{l}\text { I am confident I can understand the most complex materials that will be } \\
\text { presented by the instructor in this course. }\end{array}$ \\
\hline & & $\begin{array}{l}\text { I am confident I can do an excellent job on the assignments and tests in this } \\
\text { course. }\end{array}$ \\
\hline & 23. & I expect to do well in this class. \\
\hline & 29. & I am certain I can master the skills that will be taught in this course. \\
\hline & 30 . & $\begin{array}{l}\text { Considering the difficulty of this course, the teacher, and my skills, I think } \\
\text { I will do well in this course. }\end{array}$ \\
\hline
\end{tabular}

